\title{
An Analysis of Combining Methods in Cooperative Spectrum Sensing over Rayleigh Fading Channel
}

\author{
Tran Thanh Truc $\cdot$ Hyung-Yun Kong
}

\begin{abstract}
This paper evaluates the performance of two methods of spectrum sensing: the linear combining method and the selection combining method which is based on maximum SNR of sensing channel. We proposed a rule for global detection for the purpose of combating hidden terminal problems in spectrum sensing. Our analysis considers a situation when sensing channels experience the non-identically, independently distributed(n.i.d) Rayleigh fading. The average probabilities of global detection in these methods are derived and compared. In the scope of this paper, the reporting channels are assumed to be the AWGN channel with invariant and identical gain during the system's operation.
\end{abstract}

Key words : Maximum Ratio Combining, Cognitive Radio, Rayleigh Fading, Relay Selection, Cooperative Spectrum Sensing.

\section{Introduction}

Because free frequency band, new wireless communication technology must be more significantly effective than the existing licensed system in terms of spectrum usage. Recently, cognitive radio has been emerging as the key technology which is able to satisfy the strict requirements of spectrum efficiency ${ }^{[1]}$. This kind of system works as a secondary user(SU) system to enable the coexistence with licensed users or primary users. Thus, a sensing technique is required in order to allow secondary user to be aware of PU in order to avoid harmful interference. Due to multipath fading, vicinity and shadowing, detection operation is not always reliable ${ }^{[2]}$. Consequently, a cooperative model of sensing has been proposed as a method that can reduce error in making final decisions ${ }^{[3]}$. Detection operation is normally implemented in two successive stages: sensing and reporting. In the sensing stage, every SU carries out spectrum sensing individually. Then, in the reporting stage, observations of local sensing are reported to a common receiver (or fusion center) for making global decisions to determine whether PU is present or absent.

There are several techniques on which cooperative spectrum sensing is based such as: linear combining ${ }^{[4]}$, cluster division $^{[5]}$, and relay selection ${ }^{[6]}$. In [5], the reporting channel experiences Rayleigh fading, and the most favorable user with the largest reporting channel gain is selected to collect the results from other users. The se- lected users have two functions: collecting observation signals from local and then making binary decisions to forward to a common receiver. In [6], the relay selection method has been investigated in the environment of i.i.d probability distribution of Rayleigh fading. In the scenario of high SNRs fusion, [4] uses the linear combining method to optimize the performance of detection.

Relying on the model of [4] and [6], this paper considers two cooperative sensing techniques: 1) linear combining local soft-decision, 2) and secondary users selection combining based on a maximum sensing channel SNR in the environment of n.i.d Rayleigh fading. Hence, the probability density function(pdf) of the linear combining signal and SU selection methods are needed for calculation in a situation when this kind of fading occurs. We then propose a method to prevent the incorrect decision from hidden terminal problem which is normally caused by multipath fading, shadowing and vicinity.

Part II of this paper gives the theoretical derivation for the analysis of spectrum sensing performance in the two mentioned cases and then demonstrates the proposed rule of detection. Part III presents the Conclusion. Appendices A, B, C support part $\Pi$.

\section{Theory}

\section{2-1 System Model}

The system(Method I in this paper) has been des-

Manuscript received June 4, 2010 ; revised August 31, 2010. (ID No. 20100604-01J)

School of Electrical Engineering, University of Ulsan, Ulsan, Korea.

Corresponding Author : Hyung-yun Kong (e-mail : hkong@mail.ulsan.ac.kr) 
cribed by [4] in which the linear combining technique is employed(Fig. 1) in a condition of the high fusion SNR. In this paper, the sensing channel is a slow n.i.d Rayleigh fading channel with.

In [4], the PU signal $s(k)$ is modulated as the complex PSK signal and is identically and independently distributed(i.i.d.) with zero mean and the variance of $\sigma_{s}^{2} ; h_{s i}$ is the sensing channel gain, which is assumed to be constant during each cooperative spectrum sensing period and experiences slow flat Rayleigh fading; noise in sensing channel is i.i.d and follows a circular, symmetric, complex Gaussian random variable with zero mean and variance of $\sigma_{n s}^{2}$. The local sensing SNR is $\gamma_{s i}=$ $\sigma_{s}^{2}\left|h_{s i}\right|^{2} / \sigma_{n s}^{2}$.

Assuming that the number of samples taken for local energy detection is large enough $(K \gg 1)$, the ratio of the AWGN noise variance in the reporting channel $\sigma_{n r}^{2}$ and the square of the sensing noise variance $\sigma_{n s}^{4}$ can be neglected in comparison with gain of the reporting channel $g_{i}$, the sensing channel SNR at each SU is much smaller than unit $\left(r_{S i} \ll 1\right)^{[4]}$. In this paper, the channel gain $g_{i}$ is invariant and identical for all reporting channels $\left(g_{i}=g, \forall i\right)$ and is significantly larger than the reporting channel noise. Because the paper considers the situation of a slow flat fading sensing channel, the channel gain of each channel is not changed during each period of energy detection. Thus, the combining signal, and then its probability of detection can be obtained from [4] as showed in (1) and (2)

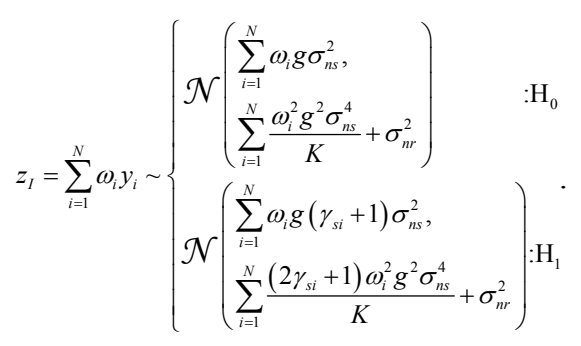

We assume the system is the high SNR fusion ${ }^{[4]}$, hence:

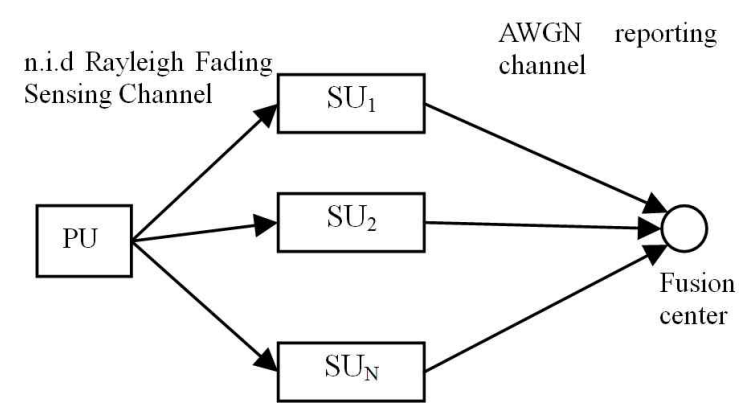

Fig. 1. System model.

$$
\begin{aligned}
P_{d}^{l} & =Q\left(Q^{-1}(\alpha)-\frac{\sum_{i=1}^{N} \omega_{i g} \gamma_{i}}{\sqrt{\sum_{i=1}^{N} \omega_{i}^{2} g^{2} / K}}\right), \\
& =Q\left(Q^{-1}(\alpha)-\frac{\sum_{i=1}^{N} \omega_{i} \gamma_{i}}{\sqrt{\sum_{i=1}^{N} \omega_{i}^{2} / K}}\right)
\end{aligned}
$$

and threshold of detection is

$$
\lambda_{I}=\sum_{i=1}^{N} \omega_{i} g \sigma_{n s}^{2}+Q^{-1}(\alpha) \sqrt{\frac{\left(2 \gamma_{s i}+1\right) \omega_{i}^{2} g^{2} \sigma_{n s}^{4}}{K}+\sigma_{n r}^{2}}
$$

where $K$ is the number of samples being taken by local energy detector, $\omega=\left[\omega_{1}, \omega_{2}, \ldots, \omega_{N}\right]^{T}$ is the linear weight vector with $\|\omega\|=1$ and $\omega_{i} \geq 0, g=g_{1}=\cdots=g_{N}$ is the reporting channel gain, $\gamma=\left[\gamma_{s 1}, \gamma_{s 2}, . ., \gamma_{s N}\right]^{T}$ is the SNR of sensing channels and $y=\left[y_{1}, y_{2}, . ., y_{N}\right]^{T}$ is the reporting signals received by fusion center. $\alpha$ is denoted as probability of false alarm, and one-dimension $Q$ function is defined as the following

$$
Q(x)=\frac{1}{2 \pi} \int_{x}^{+\infty} \exp \left(-t^{2} / 2\right) d t
$$

Being more convenient in our analysis, we rewrite the average and variance of each received signal $y_{i}$ at fusion center in the short forms as the below description:

$$
\begin{aligned}
& \mu_{y i}=\left\{\begin{array}{l}
\mu_{y_{i}, 0}=g \sigma_{n s}^{2}: H_{0}, \\
\mu_{y, 1}=g\left(\gamma_{s i}+1\right) \sigma_{n s}^{2}: H_{1},
\end{array}\right. \\
& \sigma_{y_{i}}^{2}=\left\{\begin{array}{l}
\sigma_{y_{i}, 0}^{2}=g^{2} \sigma_{n s}^{4} / K+\sigma_{n r}^{2}: H_{0} \\
\sigma_{y_{j}, 1}^{2}=\left(1+2 \gamma_{s i}\right) g^{2} \sigma_{n s}^{4} / K+\sigma_{n r}^{2}: H_{1}
\end{array} .\right.
\end{aligned}
$$

Unlike Method I, Method II uses the technique of SU selection based on the best SNR sensing channel. Rather than combining observations from all SUs, the fusion center chooses the SU that possesses the best SNR of the sensing channel to dispatch its soft-decision. Hence, the probability of detection in this case is the special case of (1) when $N=1, \omega_{i}=1$. The statistics of the received signal for this case are showed in (8) where $\hat{i}$ denotes as selected SU:

$$
\begin{aligned}
& \hat{i}=\arg \max \left\{\gamma_{s i}\right\}_{i=1}^{N}, \\
& z_{I I}=y_{s i} \sim\left\{\begin{array}{c}
\mathcal{N}\left(g \sigma_{n s}^{2}, \frac{g^{2} \sigma_{n s}^{4}}{K}+\sigma_{n r}^{2}\right): H_{0} \\
\mathcal{N}\left(\begin{array}{l}
g\left(\gamma_{s i}+1\right) \sigma_{n s}^{2}, \\
\frac{\left(2 \gamma_{s i}+1\right) g^{2} \sigma_{n s}^{4}}{K}+\sigma_{n r}^{2}
\end{array}\right): H_{1}
\end{array}\right.
\end{aligned}
$$

The probability of detection for Method $\Pi$ (maximum 
SNR based selection method) is a special case of (2). Hence, we can achieve:

$$
P_{d}^{I I}=Q\left(Q^{-1}(\alpha)-\gamma_{\hat{i}} \sqrt{K}\right)
$$

with the threshold of detection as

$$
\begin{aligned}
\lambda_{I I}=g \sigma_{n s}^{2} & +Q^{-1}(\alpha) \times \\
& \times \sqrt{\frac{\left(2 \gamma_{s i}+1\right) g^{2} \sigma_{n s}^{4}}{K}+\sigma_{n r}^{2}} .
\end{aligned}
$$

\section{2-2 Proposed Detection Rule}

From (1) and (8), we have

$$
w=z_{I}-z_{I I}=\left(w_{\hat{i}}-1\right) y_{\hat{i}}+\sum_{i=1, i \neq \hat{i}}^{N} w_{i} y_{i} .
$$

Hence, we achieve:

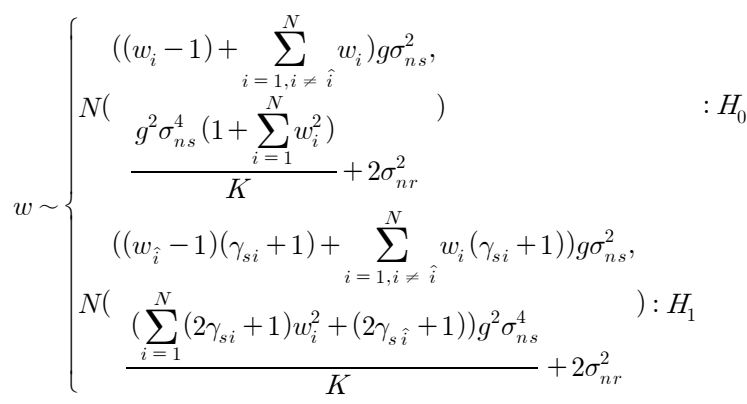

The region of the detection rule is described in Table 1. The signal $\|w\|$ indicates the distance between the combining signal $z_{I}$ and the selecting signal $z_{I I}$. Whenever $z_{I}$ is below its threshold $\lambda_{I}$, the fusion center checks the difference between two kinds of the received signals. If the difference in this case is smaller than a given $\varepsilon_{P}$, there is a decision of ' 0 ' which presents the state of no PU user being made. In the case that is affected by hidden terminal phenomenon, PU is still on active while the final decision is ' 0 ', which denotes the absence of PU. Thus, in this case, the fusion center's detection is a miss detection. Otherwise, if there is really no PU on active $\left(H_{0}\right)$, this decision is true to denote the available channel for SUs to use. The abandoned region is reserved for censoring uncertainty related effects.

From appendix C, we obtain the joint pdf of the pair of random variables $\left(z_{I}, w\right)$, as showed in (13)

$$
\begin{aligned}
& \left(z^{I}, w\right) \sim\left\{\begin{array}{l}
\mathcal{N}\left(\mu_{z_{0}^{\prime}}, \mu_{w_{0}} ; \sigma_{z_{0}^{l}}, \sigma_{w_{0}} ; r_{0}\right): H_{0} \\
\mathcal{N}\left(\mu_{z_{1}^{l}}, \mu_{w_{1}} ; \sigma_{z_{1}^{l}}, \sigma_{w_{1}} ; r_{1}\right): H_{1}
\end{array}\right. \\
& r=\left\{\begin{array}{l}
w^{T} R_{y} \tilde{w}-g^{2} \sigma_{n s}^{4}\left(\sum_{i=1}^{N} w_{i}\right) \times\left(\left(w_{i}-1\right)+\sum_{i=1, i \neq \hat{i}}^{N} w_{i}\right)=0 \\
w^{T} R_{y} \tilde{w}-g^{2} \sigma_{n s}^{4}\left(\sum_{i=1}^{N} w_{i}\left(\gamma_{s i}+1\right)\right) \times\left(\left(w_{\hat{i}}-1\right)\left(\gamma_{s i}+1\right)+\sum_{i=1, i \neq \hat{i}}^{N} w_{i}\left(\gamma_{s i}+1\right)\right)=0 \quad: H_{1}
\end{array}\right.
\end{aligned}
$$

Table 1. Detection rule.

\begin{tabular}{|c|l|l|}
\hline Region $\backslash$ Hypothesis & \multicolumn{1}{|c|}{$\mathrm{H}_{1}$} & \multicolumn{1}{c|}{$\mathrm{H}_{0}$} \\
\hline$|w| \leq \varepsilon_{P}, z_{I} \geq \lambda_{I}$ & Detection & False alarm \\
\cline { 1 - 2 }$|w|>\varepsilon_{P}, z_{I} \geq \lambda_{I}$ & & \\
\hline$|w|>\varepsilon_{P}, z_{I}<\lambda_{I}$ & Abandoned region & Abandoned region \\
\hline$|w| \leq \varepsilon_{P}, z_{I}<\lambda_{I}$ & Miss detection & PU absence \\
\hline
\end{tabular}

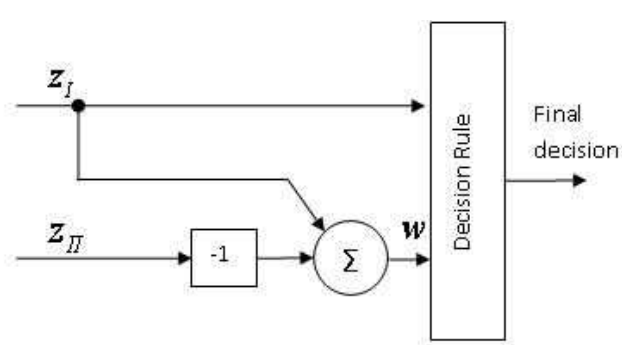

Fig. 2. Proposed detection rule.

where $\left(\mu_{z_{0}^{\prime}}, \sigma_{z_{0}^{\prime}}\right)$ and $\left(\mu_{z_{1}^{\prime}}, \sigma_{z_{1}^{\prime}}\right)$ have been determined in (1); and $\left(\mu_{w_{0}}, \sigma_{w_{0}}\right),\left(\mu_{w_{1}}, \sigma_{w_{1}}\right)$ are identified in (12). Putting $\tilde{\omega}=\left[\omega_{1}, \omega_{2}, . .,\left(\omega_{\hat{i}}-1\right), . ., \omega_{N}\right]^{T}$. Hence, we can rewrite:

$$
z_{I}=\omega^{T} y \text { and } w=\tilde{\omega}^{T} y .
$$

From appendix $\mathrm{C}$, we have the correlation coefficient of two variables $\left(z_{I}, w\right)$ :

$$
r=\omega^{T} R_{y} \tilde{\omega}-E\left\{z_{I}\right\} E\{w\} .
$$

The correlation matrix of $y$ is

$$
R_{y}=E\left\{y y^{T}\right\}= \begin{cases}R_{y, 0} & : H_{0} \\ R_{y, 1} & : H_{1}\end{cases}
$$

From (8), we can induce the correlation matrix for each SU as showed in (17), (18) and derive the value of correlation coefficient:

$$
\begin{aligned}
R_{y, 0} & =g^{2} \sigma_{n s}^{4}[1]_{N \times N} . \\
R_{y, 1} & =\left[\begin{array}{lll}
\mu_{y_{1}, 1}^{2} & . . & \mu_{y_{1}, 1} \mu_{y_{N}, 1} \\
\mu_{y_{2}, 1} \mu_{y_{1}, 1} & . . & \mu_{y_{2}, 1} \mu_{y_{N}, 1} \\
\ldots & . . & \ldots \\
\mu_{y_{N}, 1} \mu_{y_{1}, 1} & . . & \mu_{y_{N}, 1}^{2}
\end{array}\right]_{N \times N},
\end{aligned}
$$

The equation (19) permits us to conclude that these 
The threshold $\lambda_{I}$ has been identified in (3). Threshold $\varepsilon_{P}$ is selected in the requirement of a given probability of correct decision of PU absence state(Table 1). Hence,

$$
\begin{aligned}
& \beta=P_{w}\left(|w| \leq \varepsilon_{P}\right) P_{z_{I}}\left(z_{I}<\lambda_{I}\right): H_{0} \\
& \Rightarrow P_{w}\left(|w| \leq \varepsilon_{P}\right)=\frac{\beta}{1-\alpha}
\end{aligned}
$$

where $\beta$ is the probability of correct detection of $\mathrm{PU}$ absence state.

By (20), we can find the unique value of $\beta_{1}, \beta_{2}$, $\beta_{1}<\beta_{2}$ which $\beta_{1}+\beta_{2}=\frac{\beta}{1-\alpha}$. We can rewrite expression of $\varepsilon_{P}$ similar to (3). Finding $\beta_{1}, \beta_{2}$ is outside of this paper's scope.

$$
\begin{aligned}
& \varepsilon_{P}=\mu_{w, 0}+Q^{-1}\left(\beta_{2}\right) \sqrt{\sigma_{w, 0}^{2}} \\
& -\varepsilon_{P}=\mu_{w, 0}+Q^{-1}\left(\beta_{1}\right) \sqrt{\sigma_{w, 0}^{2}} .
\end{aligned}
$$

Using the same calculation as (2) which is described concretely in [4], we can induce the probability as presented in (22):

$$
\begin{aligned}
P_{m, w} & =P_{w}\left(|w| \leq \varepsilon_{P}\right) \\
& =Q\left(\frac{Q^{-1}\left(\beta_{1}\right) \sqrt{\sigma_{w, 0}^{2}}+\left(\mu_{w, 0}-\mu_{w, 1}\right)}{\sqrt{\sigma_{w, 1}^{2}}}\right)- \\
& -Q\left(\frac{Q^{-1}\left(\beta_{2}\right) \sqrt{\sigma_{w, 0}^{2}}+\left(\mu_{w, 0}-\mu_{w, 1}\right)}{\sqrt{\sigma_{w, 1}^{2}}}\right) .
\end{aligned}
$$

Under our assumption of a high SNR fusion condition as mentioned, we can make the following approximation

$$
\begin{aligned}
P_{m, w} & =P_{w}\left(|w| \leq \varepsilon_{P}\right) \\
& \approx Q\left(Q^{-1}\left(\beta_{1}\right)-\frac{\left(\sum_{i=1}^{N} \omega_{i} \gamma_{s i}-\gamma_{\hat{i}}\right)}{\sqrt{\left(\sum_{i=1}^{N} \omega_{i}^{2}+1\right) / K}}\right)- \\
& -Q\left(Q^{-1}\left(\beta_{2}\right)-\frac{\left(\sum_{i=1}^{N} \omega_{i} \gamma_{s i}-\gamma_{\hat{i}}\right)}{\sqrt{\left(\sum_{i=1}^{N} \omega_{i}^{2}+1\right) / K}}\right) .
\end{aligned}
$$

Miss detection probability is identified as (24):

$$
\begin{aligned}
H_{1}: P_{z_{I}, w} & \left(\begin{array}{l}
|w| \leq \varepsilon_{P}, z_{I}<\lambda_{I} ; \\
\mu_{z_{1}^{\prime}}, \mu_{w_{1}} ; \sigma_{z_{1}^{\prime}}, \sigma_{w_{1}} ; r_{1}
\end{array}\right) \\
= & P_{w}\left(|w| \leq \varepsilon_{P}\right) P_{z_{I}}\left(z_{I}<\lambda_{I}\right) .
\end{aligned}
$$

\section{2-3 Average Probability of Detection}

For calculating the average probability of detection, from (2), we need to derive the pdf of factor $\gamma_{Z_{t}}$ $\sum_{i=1}^{N} \omega_{i} \gamma_{i}$. Appendix A is presented to analyze the problem. Some results of specific value of $N$ are showed in the condition of i.i.d distribution of $\gamma$ in the following:

$N=2$,

$$
\begin{aligned}
f_{\gamma_{z_{I}}}(\gamma) & =\frac{\left(\omega_{1} \omega_{2}\right)^{2}}{\left(\omega_{2}-\omega_{1}\right) \bar{\gamma}} \times \\
& \times\left(\exp \left(-\frac{\gamma}{\omega_{1} \bar{\gamma}}\right)-\exp \left(-\frac{\gamma}{\omega_{2} \bar{\gamma}}\right)\right),
\end{aligned}
$$

$N=3$,

$$
\begin{aligned}
f_{\gamma_{z_{I}}}(z)= & \frac{\left(\omega_{1} \omega_{2} \omega_{3}\right)^{2}}{\left(\omega_{2}-\omega_{1}\right) \bar{\gamma}}\left\{\frac{\omega_{1}}{\omega_{1}-\omega_{3}} \exp \left(-\frac{\gamma}{\omega_{1} \bar{\gamma}}\right)-\right. \\
& -\frac{\omega_{2}}{\omega_{2}-\omega_{3}} \exp \left(-\frac{\gamma}{\omega_{2} \bar{\gamma}}\right)+ \\
& \left.+\left(\frac{\omega_{2}}{\omega_{2}-\omega_{3}}-\frac{\omega_{1}}{\omega_{2}-\omega_{3}}\right) \exp \left(-\frac{\gamma}{\omega_{3} \bar{\gamma}}\right)\right\} .
\end{aligned}
$$

For a special case when $\omega_{1}=\cdots=\omega_{N}=\omega$ for all $i=1$, $\cdots, N$ and $N \geq 2$ :

$$
f_{\gamma_{z_{I}}}(\gamma)=\frac{\gamma^{N-1}}{(N-1) !(\omega \bar{\gamma})^{N}} \exp \left(-\frac{\gamma}{\omega \bar{\gamma}}\right) \text {. }
$$

In Method $\Pi$, based on the analysis in Appendix B, the pdf of the selection method described in (7) can be derived as showed in (28).

$$
f_{\gamma_{s \hat{i}}}\left(\gamma_{s \hat{i}}\right)=\sum_{i=1}^{N} \partial \frac{F_{\gamma_{s \hat{i}}}\left(\gamma_{s \hat{i}}\right)}{\partial \gamma_{s i}}=\sum_{i=1}^{N} f_{\gamma_{\gamma_{i}}}\left(\gamma_{s \hat{i}}\right) \prod_{\substack{j=1 \\ j \neq i}}^{N} F_{\gamma_{s j}}\left(\gamma_{s \hat{i}}\right)
$$

where $f_{\gamma_{s}}\left(\gamma_{\nu^{\prime}}\right)=\frac{1}{\bar{\gamma}_{s}} \exp \left(-\frac{\gamma_{s}}{\bar{\gamma}_{s}}\right)$ is the pdf of SNR of sensing channel at the $i^{\text {th }} \mathrm{SU}$ and $F_{\gamma_{s j}}$ is its CDF function respectively.

\section{2-4 Analytical Results}

By Method I, the average value of detection probability, and miss detection probability in combining technique is evaluated by (29), (30).

$$
\begin{aligned}
& \bar{P}_{d}^{I}=\int_{0}^{+\infty} Q\left(Q^{-1}(\alpha)-\frac{\gamma_{z_{l}}}{\sqrt{\sum_{i=1}^{N} \omega_{i}^{2} / K}}\right) f_{\gamma_{z_{I}}}(\gamma) d \gamma_{z_{l}} \\
& \text { with } \gamma_{z_{t}}=\sum_{i=1}^{N} \omega_{i} \gamma_{i} \text {, and } \\
& \bar{P}_{m}^{I}=1-\bar{P}_{d}^{I}
\end{aligned}
$$


By Method II, its performance is evaluated in (31):

$$
\overline{p_{d}^{I I}}=\int_{0}^{+\infty} Q\left(Q^{-1}(\alpha)-\gamma_{s \hat{i}} \sqrt{K}\right) f_{\gamma_{s \hat{i}}}\left(\gamma_{s i}\right) d \gamma_{s i}
$$

The miss detection in Method $\Pi$ is calculated in the same way as (30).

From Table 1, we can easily see that for the proposed scheme of detection, the probability of detection does not depend on the selection method(Method II). Thus, the proposed method has the same detection probability value as the described linear combining method(Method I). However, its miss detection probability is different from Method I and. By (20), (22) and (30), it can be described as the following:

$$
\begin{aligned}
\overline{p_{m}^{I I I}}= & \int_{0}^{+\infty} \int_{0}^{+\infty}\left(1-P_{d}^{I}\right) P_{m, w} \times \\
& f_{\gamma_{Z_{I}}}\left(\gamma_{Z_{I}}\right) f_{\gamma_{s \hat{i}}}\left(\gamma_{s \hat{i}}\right) d \gamma_{z_{I}} \gamma_{s \hat{i}}
\end{aligned}
$$

The analytical results will demonstrate the performance of three methods in cases of n.i.d environment(Table 2), i.i.d fading environment with different number of users(Table 3) and i.i.d environment with the same number of users $(N=8)$ but in different $\operatorname{SNR}([-12,-14$, $15] \mathrm{dB})$. The reporting channel gain is set at $30 \mathrm{~dB}$. In addition, the number of samples taken for energy detection is 600 .

Fig. 3. demonstrates the performance of global detection when the number of SUs is kept constant at value of $N=4$ and the condition of fading is n.i.d fading. From this plot, we can see that in the SNR2 set, the selection method is better than the other two methods(the linear combining and proposed methods). Meanwhile, it is worse (than the others) in condition of the SNR1 set. We also noted that the probability of detection in the proposed scheme is the same as the combining method.

Table 2. Parameter of analysis of the case when the number of user is 4 , and n.i.d fading environment.

\begin{tabular}{|c|c|}
\hline Number of SUs & $N=4$ \\
\hline Average SNR & SNR 1 $=[-10,-13,-14,-16] \mathrm{dB}$ \\
SNR 2 $=[-10.5,-11,-10,-12] \mathrm{dB}$ \\
\hline$w_{i}$ & $1 / 2$ \\
\hline
\end{tabular}

Table 3. Parameter of analysis of the case when average SNR is $-10 \mathrm{~dB}$, and i.i.d fading environment.

\begin{tabular}{|c|c|}
\hline Number of $\operatorname{SUs}(N)$ & {$[4,8,12]$} \\
\hline Average SNR & {$[-10] \mathrm{dB}$} \\
\hline$w_{i}$ & $1 / \sqrt{N}$ \\
\hline
\end{tabular}

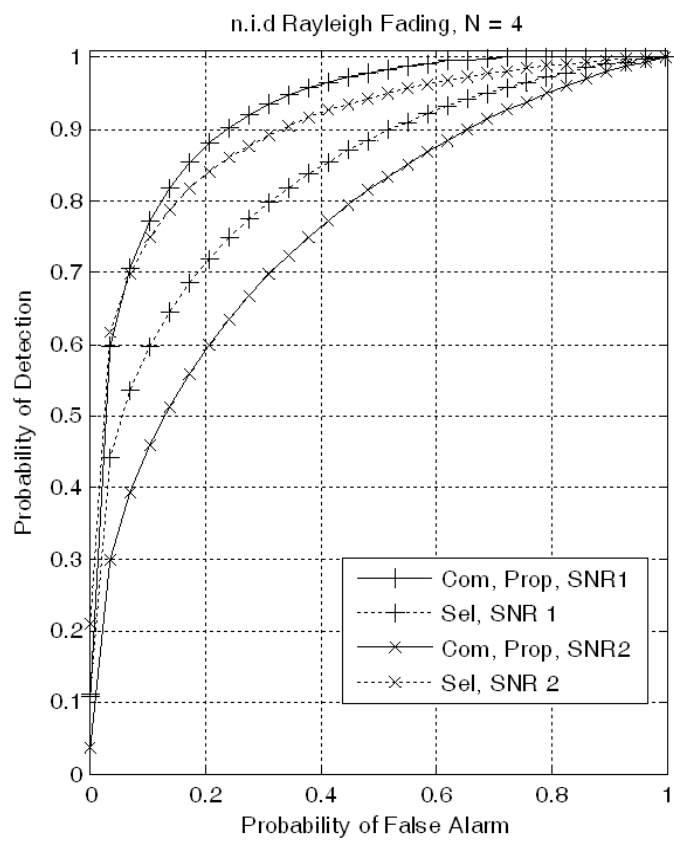

Fig. 3. Average probability of detection vs false alarm probability when $N=4$, n.i.d fading with two SNR sets described in Table 2 .

Fig. 4 provides us the performance when fusion misses PU detection. This shows that there are few differences between the proposed scheme and the others in terms of preventing miss detection under n.i.d fading environment, in the SNR1 set. It probably depends on the SNR set that we choose to survey.

Fig. 5 7 investigate the cases of i.i.d environment. Within the same number of users $(N=8$ in this case) but at a different average SNR value of Rayleigh fading, Fig. 5 shows that the detection performance of Method I and the proposed method is better than the selection

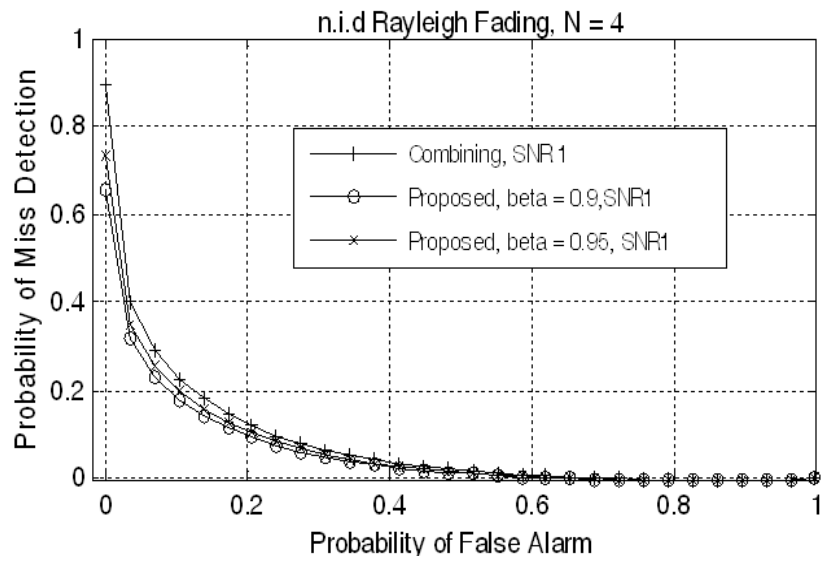

Fig. 4. Probability of miss detection vs. false alarm probability when $N=4$ and under n.i.d Rayleigh fading with set of SNR1 described in Table 2. 


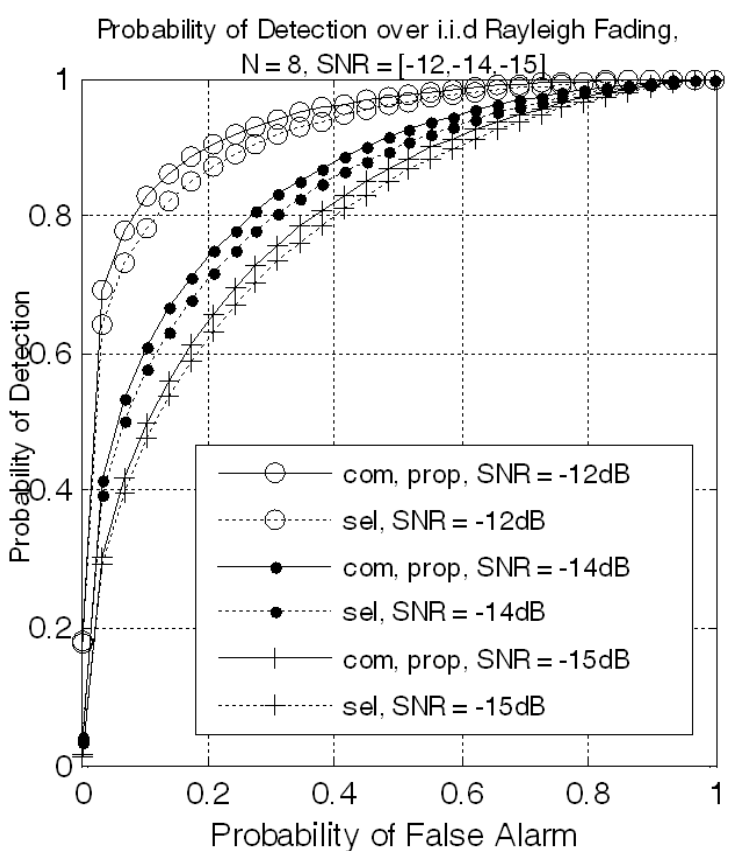

Fig. 5. Probability of detection vs. false alarm probability when $N=8$, i.i.d Rayleigh with $\mathrm{SNR}=[-12,-14,-15]$ $\mathrm{dB}$.

method. In additions, when SNRs of sensing channel are better, the detection performance is enhanced. We can see that detection probabilities in Figs. 3 and 5 are at high value even at the low sensing channel SNR. This result comes mainly from the large number of samples in our simulation parameters. Furthermore, due to the fact that the proposed scheme is still in line with the linear combining method, the detection performance is still able to exploits the diversity gain of this method. On other hand, in Fig. 6, there is also a reduction of miss detection when the proposed method instead of the combining method is used. Fig. 7 shows a survey for the case that the number of SUs is changed in the same condition as average SNR. As we can see when the number of SUs increases, the probability of miss detection decreases and therefore the proposed scheme eliminate miss detection better than the linear combining scheme.

\section{Conclusion}

In summary, this paper has considered a cooperative scheme where linear combining and the best SU selection are used for global detection in the condition of n.i.d fading environment. The paper then proposes a detection rule to eliminate miss detection. The proposed scheme has showed that it can reduce miss detection while keeping the same reliability of determining the absence state of PU(within given $\beta$ ) in condition of i.i.d Ray

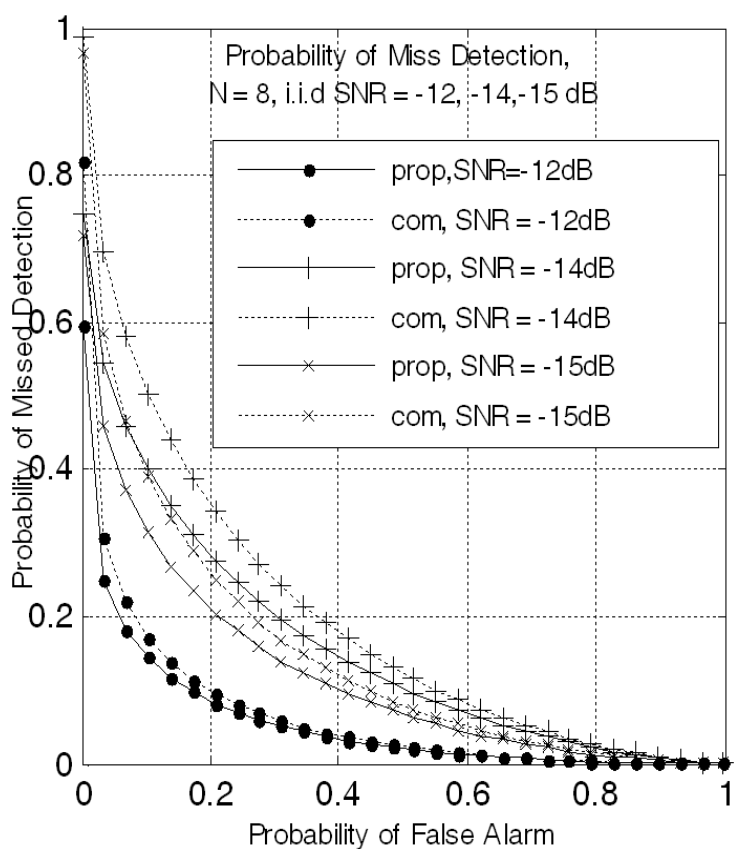

Fig. 6. Probability of miss detection vs. false alarm probability when $N=8$, i.i.d Rayleigh with $\mathrm{SNR}=[-12$, $-14,-15] \mathrm{dB}, \beta=0.8$.

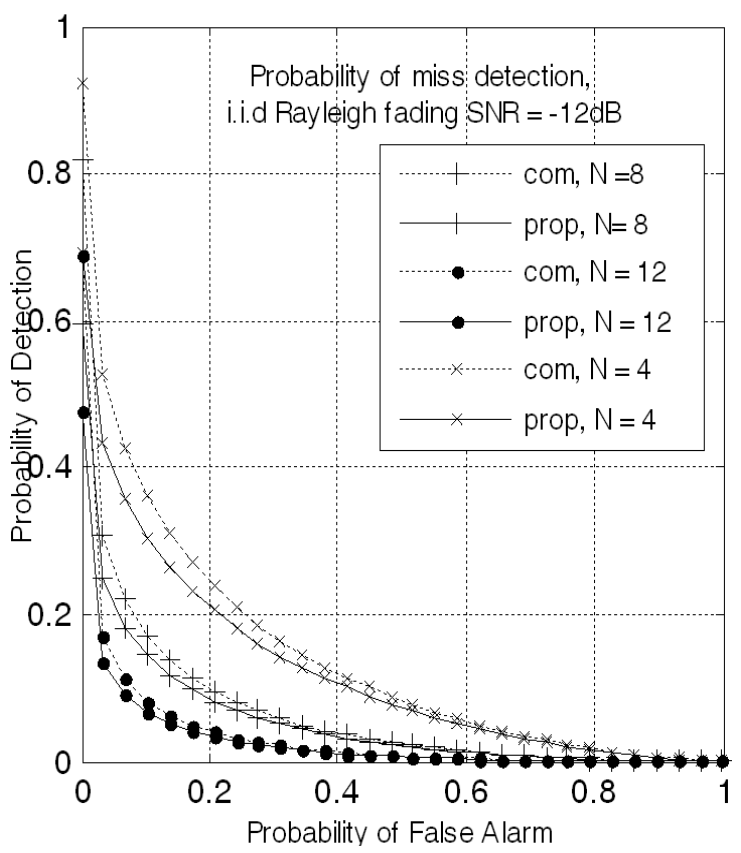

Fig. 7. Probability of miss detection vs. false alarm probability for case number of user changed and i.i.d Rayleigh fading with $\mathrm{SNR}=-12 \mathrm{~dB}, \beta=0.8$.

leigh fading. In detection operation of this fading, the linear combining method and proposed scheme have showed better performance than the selection method when they operate in i.i.d environment. In n.i.d Rayleigh fading, depending on each variance SNR of fading in 
the group of SUs, the linear combining technique's performance and proposed scheme are better or worse than the method of selection(Method II). Moreover, in environment of n.i.d Rayleigh fading, the pdf of combining random variables of SNR also has been derived to calculate average probability of detection. The performance is improved when SNRs of sensing channels improve or the number of SUs joining sensing operation increases.

\section{Appendix A}

Supposing we have vector of random variables (RVs) $\mathrm{x}=\left[x_{1}, x_{2}, . ., x_{N}\right]^{T}$, in which pdf of each element is:

$$
f_{x_{i}}\left(x_{i}\right)=\frac{1}{\bar{x}_{i}} \exp \left(-\frac{x_{i}}{\bar{x}_{i}}\right) \text {. }
$$

And combining weight $\mathrm{w}=\left[w_{1}, w_{2}, . ., w_{N}\right]^{T}$ with constraint $w_{i} \geq 0,\|w\|=1, i=1, . ., N$. Our target is to find out the pdf of this

$$
z=\mathrm{w}^{T} \mathrm{x}=\sum_{i=1}^{N} w_{i} x_{i} .
$$

Putting $y_{i}=w_{i} x_{i}$, thus the pdf of $y_{i}$ becomes:

$$
f_{y_{i}}\left(y_{i}\right)=\frac{f_{x_{i}}\left(x_{i}\right)}{\left|\partial y_{i} / \partial x_{i}\right|}=\frac{1}{w_{i} \bar{x}_{i}} \exp \left(-\frac{y_{i}}{w_{i} \bar{x}_{i}}\right)
$$

with $\lambda_{i}=\frac{1}{w_{i} \bar{x}_{i}}$. In addition, the MGF function of $y_{i}$ is:

$$
\operatorname{MGF}_{y_{i}}(s)=\frac{\lambda_{i}}{\lambda_{i}-s} ; s \leq \lambda_{i}
$$

From (34), We derive the MGF function of $z=\sum_{i=1}^{N} y_{i}$ as showed in (37) [7, section 2-1-3]:

$$
\begin{aligned}
\operatorname{MGF}_{z}(z) & =E_{z}\left(e^{s z}\right) \\
& =\int_{0}^{+\infty} . . \int_{0}^{+\infty} e^{s \sum_{i=1}^{N} y_{i}} f_{y_{1}, \ldots, y_{N}}\left(y_{1}, . ., y_{N}\right) d y_{1} d y_{2} . . d y_{N} \\
& =\int_{0}^{+\infty} e^{s y_{1}} f_{y_{1}}\left(y_{1}\right) d y_{1} \int_{0}^{+\infty} \ldots \int_{0}^{+\infty} e^{s y_{n}} f_{y_{n}}\left(y_{n}\right) d y_{n} \\
& =\prod_{i=1}^{N} \operatorname{MGF}_{y_{i}}(s)=\prod_{i=1}^{N} \frac{\lambda_{i}}{\lambda_{i}-s}
\end{aligned}
$$

We can reform the product $\prod_{i=1}^{N} \frac{\lambda_{i}}{\lambda_{i}-s}$ to the form of summation:

$$
\prod_{i=1}^{N} \frac{\lambda_{i}}{\lambda_{i}-s}=\sum_{i=1}^{N} \frac{A_{i}}{\lambda_{i}-s} ; \lambda_{i} \neq \lambda_{j} \forall i \neq j
$$

$$
\begin{aligned}
A_{i} & =\operatorname{Res}\left(\operatorname{MGF}_{z}(z), \lambda_{i}\right) \\
& =\left.\frac{\prod_{i=1}^{N} \lambda_{i}}{\partial\left(\prod_{i=1}^{N}\left(\lambda_{i}-s\right)\right) / \partial s}\right|_{s=\lambda_{i}} .
\end{aligned}
$$

Hence, MGF of $\mathrm{RV}_{\mathrm{z}}$ is

$$
\begin{aligned}
& \operatorname{MGF}_{z}(z)=\sum_{i=1}^{N} \frac{A_{i}}{\lambda_{i}-S,} \\
& f_{z}(z)=\sum_{i=1}^{N} A_{i} \exp \left(-\lambda_{i} z\right) .
\end{aligned}
$$

For the special case when $\lambda_{1}=. .=\lambda_{N}=\lambda$ for all $i=1, . . N$ and $N \geq 2: A=\lambda^{N}$

$$
\begin{aligned}
& \operatorname{MGF}_{z}(z)=\frac{A}{(\lambda-s)^{N}} \\
& f_{z}(z)=\frac{A z^{N-1}}{N-1 !} \exp (-\lambda z)
\end{aligned}
$$

\section{Appendix B}

Considering we have a vector of RVs $\mathrm{x}$ which is identified such that: $\mathrm{x}=\left[x_{1}, x_{2}, . ., x_{N}\right]^{T}$. This appendix calculates pdf of the selection function that selects the maximum values among number of independent RVs:

$$
y=\max \left\{x_{i}\right\}_{i=1}^{N} .
$$

Because $\left\{x_{i}\right\}_{i=1}^{N}$ is independent, the distribution of $y_{\text {is }}$ the following $[8$,section 6.2$]$ :

$$
\begin{aligned}
F_{y}\left(y_{0}\right) & =P_{y}\left(y \leq y_{0}\right) \\
& =P_{x_{1}, x_{2}, \ldots, x_{n}}\left(x_{1} \leq y_{0}, . ., x_{N} \leq y_{0}\right) \\
& =F_{x_{1}, x_{2}, \ldots, x_{n}}\left(y_{0}, . ., y_{0}\right) \\
& =F_{x_{1}}\left(y_{0}\right) F_{x_{2}}\left(y_{0}\right) . . F_{x_{N}}\left(y_{0}\right) \\
& =\prod_{i=1}^{N} F_{x_{i}}\left(y_{0}\right)
\end{aligned}
$$

Hence, this yields

$$
f_{y}(y)=\partial \frac{F_{y}(y)}{\partial x_{1} \partial x_{2} . . \partial x_{N}}=\sum_{i=1}^{N} f_{x_{i}}(y) \prod_{\substack{j=1 \\ j \neq i}}^{N} F_{x_{j}}(y)
$$

\section{Appendix C}

According to [6, section 6. 1], the joint pdf of two normally joint $\mathrm{RVs} X$ and $Y$ is 


$$
\begin{aligned}
& f_{X Y}(x, y)=\frac{1}{2 \pi \sigma_{X} \sigma_{Y} \sqrt{1-r^{2}}} \times \\
& \times \exp \left\{-\frac{1}{2\left(1-r^{2}\right)}\left[\frac{\left(x-\mu_{X}\right)^{2}}{\sigma_{X}^{2}}-\right.\right. \\
& \left.\left.-\frac{2 r\left(x-\mu_{X}\right)\left(y-\mu_{Y}\right)}{\sigma_{X} \sigma_{Y}}+\frac{\left(y-\mu_{Y}\right)^{2}}{\sigma_{Y}^{2}}\right]\right\}
\end{aligned}
$$

where the correlation coefficient is identified as below:

$$
\begin{aligned}
& r=E\{x y\}-E\{x\} E\{y\} . \\
& \text { If }(X, Y) \text { has zero mean: } \mu_{X}=\mu_{Y}=0, \\
& \begin{aligned}
F_{X Y}\left(x_{0}, y_{0}\right) & =P_{X Y}\left(x \leq x_{0}, y \leq y_{0}\right) \\
& =1-Q_{X, Y}\left(x_{0}, y_{0}, r\right)
\end{aligned}
\end{aligned}
$$

with $Q_{X, Y}($.$) as the two dimension Gaussian Q$-function:

$$
\begin{aligned}
& Q_{X, Y}\left(x_{0}, y_{0}, r\right)=\frac{1}{2 \pi \sqrt{1-r^{2}}} \times \\
& \times \int_{x_{0}}^{+\infty} \int_{y_{0}}^{+\infty} \exp \left[-\frac{u^{2}+v^{2}-2 r u v}{2\left(1-r^{2}\right)}\right] d v d u .
\end{aligned}
$$

Here, we calculate $r$ in the case when $x, y$ is the function of vector $\mathrm{RVs} \mathrm{z}=\left[z_{1}, z_{2}, . ., z_{N}\right]^{T}$ with:

$$
x=\sum_{i=1}^{N} a_{i} z_{i} ; \text { and } y=\sum_{i=1}^{N} b_{i} z_{i} ; a_{i}, b_{i} \in R
$$

we have:

$$
x y=z^{T} a b^{T} z
$$

where $a=\left[a_{1}, a_{2}, . ., a_{N}\right]^{T}, b=\left[b_{1}, b_{2}, . ., b_{N}\right]^{T}$

with given $a, b$ and $\left\{z_{i}\right\}_{i=1}^{N}$ being independent Gaussian distributed, we can derive the average value of $x y$ as the following:

$$
E\{x y\}=a^{T} R_{z} b
$$

where $R_{z}=E\left\{z z^{T}\right\}$.
This research was supported by Basic Science Research Program through the National Research Foundation of Korea(NRF) funded by the Ministry of Education, Science and Technology(No.2010-0004865).

\section{References}

[1] S. Haykin, "Cognitive radio: Brain-empowered wireless communication", IEEE J. Select. Areas Commun., vol. 23, no. 2, pp. 201-220, Feb. 2005.

[2] Z. Wei, R. Mallik, and K. Letaief, "Optimization of cooperative spectrum sensing with energy detection in cognitive radio networks", Wireless Communications, IEEE Transactions on, vol. 8, no. 12, pp. 57615766, 2009.

[3] D. Cabric, S. M. Mishra, and R. W. Brodersen, "Implementation issues in spectrum sensing for cognitive radios", in Proc. of Asilomar Conf. on Signals, Systems and Computers, Pacifc Grove, CA, USA, pp. 772-776, Nov. 2004.

[4] G. Xiong, S. Kishore, and A. Yener, "On low complexity cooperative spectrum sensing for cognitive networks", Computational Advances in Multi-Sensor Adaptive Processing(CAMSAP), 2009 3rd IEEE International Workshop on, pp. 145-148, Dec. 2009.

[5] S. Chunhua, Z. Wei, and K. Ben, "Cluster-based cooperative spectrum sensing in cognitive radio systems", Communications, 2007. ICC '07. IEEE International Conference on, pp. 2511-2515, Jun. 2007.

[6] S. Chen, Y. D. Alemseged, H. N. Tran, and H. Harada, "Cognitive radio sensing architecture and a sensor selection case study", Vehicular Technology Conference, 2009. VTC Spring 2009. IEEE 69th, pp. 1-5, Apr. 2009.

[7] J. Proakis, Digital Communication, $4^{\text {th }}$ Edition, Mc Graw Hill, 2002.

[8] Papoulis. A, Unnikrishna Pillai, S. Probability, Random Variables, and Stochastic Processes, $4^{\text {th }}$ Edition, McGraw-Hill, 2002. 


\section{Tran Thanh Truc}

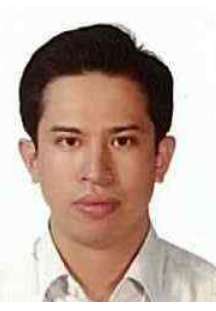

received the B.S. degrees in Telecommunication Engineering from Poly-technique University of Danang, Vietnam in 2005. In 2008, he got the degree of Master from Hochiminh University of Technology, Vietnam in major of Electrical and Electronics Engineering. Since 2010, he has been studying Ph.D. program at Univerisity of Ulsan, Korea. His major researches are Cognitive Radio Network, Cooperative Communication.

\section{Hyung-Yun Kong}

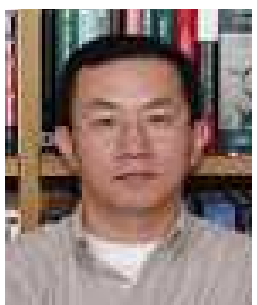

received the M.E. and Ph.D. degrees in electrical engineering from Polytechnic University, Brooklyn, New York, USA, in 1991 and 1996, respectively, He received a $\mathrm{BE}$ in electrical engineering from New York Institute of Technology, New York, in 1989. Since 1996, he has been with LG electronics Co., Ltd., in the multimedia research lab developing PCS mobile phone systems, and from 1997 the LG chairman's office planning future satellite communication systems. Currently he is a Professor in electrical engineering at the University of Ulsan, Korea. His research area includes channel coding, detection and estimation, cooperative communications, cognitive radio and sensor networks. He is a member of IEEK, KICS, KIPS, IEEE, and IEICE. 